\title{
A Comparative Analysis of Anxiety and Depression among Glaucoma and Cataractous Patients in Southwest Nigeria
}

\author{
A. C. Okudo ${ }^{1 *}$, O. E. Babalola ${ }^{2}$, A. T. Ogunro ${ }^{1}$ \\ ${ }^{1}$ Eye Foundation Hospital, Abuja, Nigeria \\ ${ }^{2}$ Rachel Eye Center, Garki, Abuja, Nigeria \\ Email: *adaoraokudo@gmail.com
}

How to cite this paper: Okudo, A.C., Babalola, O.E. and Ogunro, A.T. (2021) A Comparative Analysis of Anxiety and Depression among Glaucoma and Cataractous Patients in Southwest Nigeria. Open Journal of Ophthalmology, 11, 105-133. https://doi.org/10.4236/ojoph.2021.112009

Received: March 6, 2021

Accepted: May 11, 2021

Published: May 14, 2021

Copyright $\odot 2021$ by author(s) and Scientific Research Publishing Inc. This work is licensed under the Creative Commons Attribution International License (CC BY 4.0).

http://creativecommons.org/licenses/by/4.0/

\begin{abstract}
Background: Glaucoma is the leading cause of irreversible blindness worldwide. An appreciable proportion of glaucoma patients have been found to have hidden psychiatric disorders that often go undetected. Anxiety and depression have been found to be major contributors to these psychological issues. This present study aims to compare the prevalence of anxiety and depression amongst glaucoma and cataract patients and to identify risk factors for anxiety and depression amongst these patients. Materials and Methods: A comparative crossectional study was carried out among 197 Primary Open Angle Glaucoma (POAG) patients and 197 controls (non-POAG patients that have no ocular disorder other than cataract) attending Deseret Community Vision Institute, Ijebu Imushin, Ogun state from 1st of April to 31st May 2014. POAG was defined as patients with open anterior chamber angles based on gonioscopy, pale and cupped discs (Cup: Disc ratio $\geq 0.4$ ), and corresponding visual field defects with or without an elevated intraocular pressure. Cataract was defined as patients with opacification of the crystalline lens in either or both eyes. The Hospital Anxiety and Depression Scale (HADS) was used to assess anxiety and depression among the participants. Data were analyzed using SPSS Version 20 using Chi-square, Independent student T-test and Logistic regression. Results: The mean age of glaucoma subjects was 62.24 years and the cataract subjects was 64.2 years. There was a male preponderance with a male: female ratio of $1.5: 1$. Anxiety as defined by the HADS was seen in $35.5 \%$ of glaucoma patients and $21.8 \%$ of the cataract patients but there was no statistically significant difference after controlling for sociodermographic and clinical characteristics on logistic regression. The risk factors for anxiety among the glaucoma participants were those younger than 60 (OR 2.9, 95\% CI: $1.3-6.5 \mathrm{p}=0.009$ ) and those with severe glaucoma (OR 9.5, 95\%
\end{abstract}


CI: $1.7-54.4 \mathrm{p}=0.011)$. The risk factors for anxiety among cataract patients were sociodermographic factors. Those employed (OR 6.4. 95\% CI: $2.1-20.0$ $\mathrm{p}=0.001$ ) and those separated and divorced (OR 10.3, 95\% CI: $1.4-78.4 \mathrm{p}=$ 0.002). Visual status was not a risk factor amongst the cataract participants. Depression as defined by HADS was seen in $24.4 \%$ of glaucoma participants and $3.6 \%$ of cataract participants. Glaucoma participants were four times more likely to be depressed than the cataract participants after controlling for clinical and sociodermographic variables (OR 4.0, 95\% CI: $1.5-10.8 \mathrm{p}=$ 0.007). The risk factors for depression among the glaucoma participants were those younger than 60 (OR 4.7, 95\% CI: $1.7-13.5 \mathrm{p}=0.004)$, those with primary (OR 6.6, 95\% CI: 1.5 - 28.4) p = 0.010) and secondary education (OR 8.0, 95\% CI: $2.1-29.9 \mathrm{p}=0.002$ ) as compared to those with tertiary education, those unemployed (OR 2.0, 95\% CI: $1.0-7.2 \mathrm{p}=0.042$ ) as compared to those employed. Those blind (OR 10.8. 95\% CI: $2.8-42.4 \mathrm{p}=0.001$ ) as compared to those without visual impairment and those that had had surgery (OR 3.7, 95\% CI: $1.4-10.0 \mathrm{p}=0.011)$. Conclusion: Anxiety and Depression were found in $35.5 \%$ and $24.4 \%$ of glaucoma patients as compared to $21.8 \%$ and $3.6 \%$ of cataract patients respectively. Glaucoma patients were 4 times more likely to be depressed than patients with cataracts. There was no significant difference in anxiety between the two groups but glaucoma patients had a four times higher risk of being depressed as compared to cataract patients. There is a need to address these psychological issues at the community level in order to improve the quality of life of these patients. It is also important to identify those at risk in order to curb this growing trend/concern.

\section{Keywords}

Anxiety, Depression, Glaucoma, Cataract

\section{Introduction}

Glaucoma is the leading cause of irreversible blindness worldwide and in Nigeria [1] [2]. Worldwide there are 66,800,000 people affected with glaucoma of which $10 \%$ are bilaterally blind [1]. In Nigeria 147,000 individuals are visually impaired and blind from glaucoma and it accounts for $16.7 \%$ of blindness [2]. Being diagnosed to have glaucoma (the leading cause of irreversible blindness), the intensive lifelong management consisting of periodic follow-up visits, investigations, use of medications and surgery has been found to elicit anxiety and depression amongst glaucoma patients [3]-[10]. An appreciable proportion of glaucoma patients have been found to have hidden psychiatric disorders that often go undetected. Anxiety and depression have been found to be major contributors to these psychological issues [3]-[10].

There have been mixed reports on anxiety and depression in glaucoma patients. Some show that anxiety and depression are not significantly more in glaucoma patients than others [3] [4] [5] while other reports have shown that 
these psychological issues are of rising concern in glaucoma patients than non glaucomatous patients [6] [7] [8] [9] [10]. Weiss et al. reported that $21.1 \%$ of Israeli glaucoma clinic patients had depression but this was similar to the general Israeli population [3]. Wilson et al. also found $9.5 \%$ of glaucoma patients and $4.8 \%$ of glaucoma suspects as compared to $21.4 \%$ of non glaucoma patients had depression [4]. Amongst glaucoma clinic patients attending University of Benin Teaching Hospital (UBTH) 10\% were found to be anxious and 6\% were depressed and these were not different from the normal population [5]. While Mitsonis et al. found depression to be more prevalent amongst Greek glaucoma clinic patients than non glaucoma patients [6]. Akindipe et al. also reported that $22 \%$ of glaucoma patients had depression as compared to $11 \%$ of cataract patients [7]. Okudo reported that $25.5 \%$ of glaucoma clinic patients in Abuja had depression as compared to $8.5 \%$ of patients without any visual impairing ocular disorder and $17.9 \%$ had major depression as compared to $2.8 \%$ of the non glaucoma patients [8]. Erb et al. also reported that depression was higher in glaucoma patients than controls [9]. Tastan et al. also that reported 14\% of Turkish patients to have depression and 57\% to be anxious [11]. Zhou et al. reported $22.92 \%$ of Chinese glaucoma clinic patients had depression and $16.4 \%$ were anxious [12].

It is of utmost importance that anxiety and depression are studied amongst glaucoma patients in order to identify the prevalence of these disorders amongst glaucoma patients. It will also help to determine if it is more prevalent amongst glaucoma patients than non glaucoma patients that have cataracts. This will help identify patients at risk of these disorders in order to plan strategies to prevent and manage them. Also anxiety and depression have been found to be associated with poor medication use [3] [13] [14] hence the risk of progression of the disease and blindness. It has also been associated with reduced quality of life [7] [8] [11] [12] hence managing these psychological disorders will also improve the quality of life.

Anxiety and depression are two common responses to a glaucoma diagnosis and it is important for ophthalmologist to identify and manage these issues. Ophthalmologists have also been found to create anxiety and depression amongst their patients due to failure in adequate and appropriate communication with the patient [15]. They have also presumed that these are mental health issues that should be handled by mental health specialist since they are not primarily trained to do these [15]. The patient on the other hand, seeks for answers to these problems elsewhere and is often misguided by information from the internet and the public [15].

There are a few studies on anxiety and depression amongst glaucoma patients in the country. Akindipe et al. looked at depression amongst glaucoma patients attending Lagos University Teaching Hospital (LUTH) and compared with cataract patient using the Zung Self Rating Depression scale. He found depression was more prevalent amongst glaucoma patients as compared to cataract patients 
that were similarly visually impaired [7]. Dawodu et al. on the other hand looked at depression amongst glaucoma patients attending UBTH using the Hospital anxiety and depression scale and found that the prevalence of depression was not different from that of the normal population [5]. Okudo looked at depression in patient attending two ophthalmic centers in Abuja using the Center for Epidemiological Studies on Depression and compared this with patients with no visual impairment and found glaucoma patients to be four times more likely than patients without any visual impairing ocular disorder to be depressed [8]. Most of these studies that have been carried out were in big cities and in University Teaching Hospitals. This study, on the contrary, intends to determine the prevalence of anxiety and depression amongst glaucoma patients attending a community eye hospital within a rural area with a different sociodermographic profile from the other studies which were in urban areas. Also it is a community eye hospital as compared to other studies that were carried out in teaching hospitals. It is important to observe if a different picture will arise in this setting in order to plan interventions, taking note that community eye centers do not have the multidisciplinary specialties seen in teaching hospitals and also to proffer solutions that are unique to the particular environment. The study also intends to compare anxiety and depression with cataract patients that are also at risk of being visually impaired and determine risk factors of anxiety and depression among glaucoma and cataract patients.

\section{Materials and Methods}

Study design: The study was a comparative crossectional study carried out from 1st of April to 31st May 2014.

Study population: Glaucoma and cataract patients.

Study location: Deseret Community Vision Institute (DCVI) Ijebu-Imushin, Ogun State.

Sampling Technique: Every consecutive patient with glaucoma and equal number of non glaucoma patients without any ocular morbidity other than cataract

Null hypothesis: Glaucoma Patients are not more anxious and depressed than cataract patients.

Ethical approval: The study adhered to the tenets of declaration of Helsinki [16]. Ethical approval was obtained from Federal Medical Center, Abeokuta Health Research Ethics Committee (Appendix 1) and Eye Foundation Hospital Research Ethics Committee (Appendix 2). Permission was sought from the Chief Medical Director. Each participant that agreed to participate read the patients participation sheet (Appendix 3) and signed a consent form (Appendix 4).

Sample size calculation:

The formula for comparison of two means used in this study [17] =

$$
\frac{(u+v)^{2}\left(\sigma_{1}^{2}+\sigma_{1}^{2}\right)}{\left(\mu_{1}-\mu_{0}\right)^{2}}
$$


where: $u=$ standard one sided percentage point of the normal distribution corresponding to $100 \%$ i.e. the desired power of the study. This is $95 \%$ which corresponds to 1.64 .

$V=$ percentage point of the normal distribution corresponding to the two sided significance level. E.g. for a significance level of $5 \%, v=1.96$.

$\sigma_{1}=$ standard deviation of the depression/anxiety scores in group 1.

Standard deviation of depression score of glaucoma patients seen in LUTH [7] $=6.82$.

$\sigma_{0}=$ standard deviation of the depression/anxiety scores in group 2.

Standard deviation of depression score of cataract patients seen in LUTH [7] $=6.33$.

$\left(\mu_{1}-\mu_{0}\right)=$ the anticipated mean depression score difference of the two groups $=2.5$.

\section{Therefore:}

$$
\begin{aligned}
& \frac{(u+v)^{2}\left(\sigma_{1}^{2}+\sigma_{1}^{2}\right)}{\left(\mu_{1}-\mu_{0}\right)^{2}} \\
& =\frac{(1.64+1.96)^{2}\left((6.82)^{2}+(6.33)^{2}\right)}{(2.5)^{2}} \\
& =179.53
\end{aligned}
$$

Plus $10 \%$ for attrition $=197$.

Hence 197 glaucoma and 197 cataract participants.

Inclusion criteria for the glaucoma participants

- POAG patients with

o At least 18 years of age;

o Known glaucoma patients for at least 6 months;

o Willing to participate.

Exclusion criteria for glaucoma participants

- POAG patients

o Less than 18 years;

o Less than 6 months of diagnosis of glaucoma;

o Any other form of optic neuropathy other than glaucoma;

o Any other ocular morbidity other than glaucoma;

o Known systemic co-morbidity i.e. Hypertension, Diabetes mellitus, Asthma, Migraine, Sickle cell disease, Arthritis, Kidney disease, Thyroid disease, Stroke/ cerebro-vascular accident, cardiac disease, cancer, Human Immunodeficiency Virus Syndrome and other major systemic problems.

\section{Inclusion criteria for cataract participants}

- Cataract patients attending DCVI

o Older than 18 years of age;

o With vertical cup to disc ratio $(\mathrm{VCD})<0.4$;

o Willingness to participate.

Exclusion criteria for cataract participants 
- Cataract patients

o Less than 18 years of age;

o Not willing to participate;

o Other ocular morbidities other than cataract;

o With vertical cup to disc ratio (VCD) $>0.4$;

o Known systemic co-morbidity i.e. Hypertension, Diabetes mellitus, Asthma, Migraine, Sickle cell disease, Arthritis, Kidney disease, Thyroid disease, Stroke/cerebro-vascular accident, cardiac disease, cancer, Human Immunodeficiency Virus Syndrome and other major systemic problems.

\subsection{Data Collection}

Patient selection: The researcher screened consecutive patients attending DCVI to identify patients that fit the requirements for eligible participants and controls. The screening carried out involved taking a detailed history and ocular examination. Examination carried out include unaided and aided visual acuity using Snellen's chart which was then converted to LogMAR (Appendix 5), slit lamp examination of anterior segment, gonioscopy, dilated fundoscopy with 78 $\mathrm{D}$ assessment of the optic nerve and central visual field using the frequency doubling technique visual field analyzer (Swedish Interactive threshold algorithm Standard 24-2 strategy). Details of the study were explained to the selected participants and permission was sought and a written consent was obtained.

Information concerning their age, sex, education, employment status, occupation, marital status, religion, duration of glaucoma, family history of glaucoma and blindness, and the form of treatment they were receiving was obtained.

The visual acuity, vertical cup to disc ratio, mean deviation, glaucoma severity, was noted (Appendix 6). Hospital Anxiety and Depression Scale (HADS) questionnaire (Appendix 6) was used to assess anxiety and depression in the study. It is a simple and reliable instrument for detecting states of anxiety and depression in a hospital medical outpatient clinic. It was designed by Zigmond and Snaith in 1983. It comprises of 14 questions, 7 questions to assess anxiety and 7 questions to assess depression. Each question has a four point response $(0-3)$, hence scores a range from 0 - 21 for anxiety and $0-21$ for depression. Initially scores were classified as $0-7$ normal, $8-10$ as borderline and greater or equal to 11 as a case of either anxiety or depression. Presently it is classified as $0-7$ as normal, 8 - 10 as mild, 11 - 14 as moderate and 15 - 21 as severe (Appendix 7). The questionnaire takes 2 - 5 minutes to administer [18].

The English and Yoruba version of the questionnaire was used. The questionnaire was translated in Yoruba and back translated to English to ascertain that the actual meaning of the questionnaire was maintained.

\subsection{Definition of Terms}

Primary open-angle glaucoma patients in the study was defined as patients with gonioscopically open anterior chamber angles, glaucomatous optic nerve head 
changes (vertical cup to disc ratio of greater than or equal to 0.4 with violation of the ISNT rule or disc asymmetry of greater than 0.2 ) and corresponding visual field defects in the absence of other identifiable causes. Elevation of intra-ocular pressure will not be considered in this definition [19] [20].

Severity of glaucoma in the study was classified based on both structural and functional evidence of glaucomatous optic nerve damage.

The structural evidence of damage was based on dilated stereoscopic optic nerve head examination at the slit lamp using +78 DS lens. Mild or early glaucomatous damage was defined as a vertical cup to disc ratio of 0.4 to 0.5 with violation of the ISNT rule. Moderate glaucomatous damage was defined as 0.6 to 0.7 and severe was defined as eye with cup to disc ratio greater than or equal to 0.8 in the worse eye [21].

The functional evidence of damage was classified based on Hodapp Parish and Anderson classification of the visual field deficit. Mild or early visual field defects was defined as mean deviation of less than or equal to $-6 \mathrm{~dB}$ of the worse eye, moderate visual field defects was defined as mean deviation of greater than $-6 \mathrm{~dB}$ to $-12 \mathrm{~dB}$ of the worse eye and severe was defined as mean deviation of greater than $-12 \mathrm{~dB}$ of the worse eye or participants that cannot satisfactorily complete or carry out a visual field testing because of poor visual function due to glaucoma [22].

Cataract was defined as opacification of the crystalline lens in at least one eye.

Blindness was defined as visual acuity of less than $3 / 60$ or corresponding visual field loss of less than 10 degrees in the better eye with best possible correction [23].

\subsection{Pre Survey Activities}

Training session for the two research assistants on administering the questionnaire was held at the Deseret Community Vision Institute (DCVI) on a daily basis for a week.

A pilot study was carried in DCVI before the main study. Participants were glaucoma and cataract patients attending the clinic. All participants of the study were exempted from the main study. The questionnaire was then adjusted based on findings from the study. The reliability of the instruments and the results was evaluated by assessing inter observer and test retest reliability of the visual acuity and the administration of the questionnaires.

\section{Data entry and statistical analysis:}

Data entry, editing and analysis were done using SPSS (Software Programme for Social Sciences version 20). Categorical variables like age range, sex, employment status was presented as frequencies and percentages, differences between cases and controls were compared using chi-square test. Continuous variables such as the Hospital Anxiety and Depression scores were presented as means and standard deviation and independent student $\mathrm{T}$ test was used to compare between cases and controls. Logistic regression was used to determine the 
odds of having anxiety and depression amongst the glaucoma patients and to identify independent risk factors.

\section{Results}

Three hundred and ninety four participants were enrolled into the study comprising of 197 glaucoma patients and 197 cataract patients.

Majority of the enrolled participants were males $(60.4 \%$ of glaucoma and $61.9 \%$ of the cataract participants) and above the age of 60 years $(57.4 \%$ of glaucoma and $62.9 \%$ of cataract participants). There was no statistically significant difference between the age and sex of both groups. The glaucoma participants were more educated than the cataract participants. As $21.3 \%$ of the cataract participants had not received any formal education as compared to $12.2 \%$ of the glaucoma participants. $25.9 \%$ of the glaucoma participants had tertiary education as compared $17.8 \%$ of the cataract participants. These were statistically significant. Most of the glaucoma participants $(42.1 \%)$ were retired and majority (45.2\%) of the cataract participants were self employed. There was a statistically significant difference between those employed and self employed as compared to those not actively employed.

Majority of the glaucoma and cataract patients were married. There was a statistically significant difference in the religion of both groups as majority of glaucoma participants were Christians and the cataract participants were Muslims (Table 1).

Sixty four percent of the cataract participants were visually impaired as compared to $59.9 \%$ of glaucoma participants. Although more of the glaucoma participants (45.7\%) were blind as compared to $8.1 \%$ of the cataract participants. In using only visual acuity to define the visual status $22.3 \%$ of glaucoma participants were blind as compared to $8.1 \%$ of cataract participants. Almost all the glaucoma participants (94.4\%) were on medical therapy and $84.8 \%$ were using B blockers. Twenty nine percent had had trabeculectomy. Twenty percent were aware of a family history of glaucoma. Over $3 / 4$ of the glaucoma participants had severe glaucoma and less than $10 \%$ had mild glaucoma (Table 2).

Anxiety was found to occur more in glaucoma and cataract participants than depression, $35.5 \%$ of glaucoma participants as compared to $21.8 \%$ of cataract participants were found to have anxiety. Twenty four percent of glaucoma participants were found to have depression as compared to $3.6 \%$ of cataract participants (Table 3/Figure 1).

Most of the variants of these psychological disorders were mild as $15.7 \%$ of glaucoma and $14.7 \%$ of cataract participants had mild anxiety (Table $4 /$ Figure 2). $12.7 \%$ of glaucoma and $3.6 \%$ of cataract participants had mild depression (Table 4/Figure 3). None of the cataract participants had moderate or severe depression. Mild, moderate and severe anxiety were more in glaucoma participants as compared to cataract participants and this was statically significant (Table 4/Figure 2). 
Table 1. Sociodemographic characteristics of enrolled participants.

\begin{tabular}{|c|c|c|c|c|}
\hline $\begin{array}{c}\text { Sociodemographic } \\
\text { Characteristics }\end{array}$ & $\begin{array}{c}\text { Glaucoma } \\
\text { participants }\end{array}$ & Cataract participants & $\begin{array}{c}\text { All } \\
\text { Participants }\end{array}$ & $\mathrm{p}$ value \\
\hline \multicolumn{5}{|l|}{ Sex } \\
\hline Males & $119(60.4)$ & $122(61.9)$ & $241(62.2)$ & \multirow[t]{2}{*}{0.756} \\
\hline Females & $78(39.6)$ & $75(38.1)$ & $153(38.8)$ & \\
\hline \multicolumn{5}{|l|}{ Age } \\
\hline$\leq 40$ & $18(9.1)$ & $10(5.1)$ & $28(7.1)$ & \multirow{4}{*}{0.373} \\
\hline $41-60$ & $66(33.5)$ & $63(32.0)$ & $129(32.7)$ & \\
\hline $61-80$ & $100(50.8)$ & $107(54.3)$ & $207(52.5)$ & \\
\hline$>80$ & $13(6.6)$ & $17(8.6)$ & $30(7.6)$ & \\
\hline Mean Age & $62.24 \pm 15.75$ & $64.20 \pm 13.42$ & $63.22 \pm 14.64$ & 0.183 \\
\hline \multicolumn{5}{|l|}{ Education } \\
\hline Nil & $24(12.2)$ & $42(21.3)$ & $66(16.8)$ & \multirow{4}{*}{$0.015^{\star}$} \\
\hline Primary & $64(32.5)$ & $75(38.1)$ & $139(35.3)$ & \\
\hline Secondary & $58(29.4)$ & $45(22.8)$ & $103(26.1)$ & \\
\hline tertiary & $51(25.9)$ & $35(17.8)$ & $86(21.8)$ & \\
\hline \multicolumn{5}{|l|}{ Employment } \\
\hline Employed & $28(14.2)$ & $33(16.8)$ & $61(15.5)$ & \multirow{5}{*}{0.278} \\
\hline Self employed & $75(38.1)$ & $89(45.2)$ & $164(41.6)$ & \\
\hline Unemployed & $5(2.5)$ & $5(2.5)$ & $10(2.5)$ & \\
\hline Retired & $83(42.1)$ & $68(34.5)$ & $151(38.3)$ & \\
\hline student & $6(3.0)$ & $2(1.0)$ & $8(2.0)$ & \\
\hline \multicolumn{5}{|l|}{ Employment } \\
\hline Employed/self employed & $103(52.3)$ & $124(62.9)$ & $227(57.6)$ & \multirow[t]{2}{*}{$0.032^{*}$} \\
\hline others & $94(47.7)$ & $73(37.1)$ & $167(42.4)$ & \\
\hline \multicolumn{5}{|l|}{ Marital status } \\
\hline Single & $11(5.6)$ & $4(2.0)$ & $15(3.8)$ & \multirow{4}{*}{0.246} \\
\hline Married & $134(68.0)$ & $142(72.1)$ & $276(70.1)$ & \\
\hline Widowed & $43(21.8)$ & $45(22.8)$ & $88(22.3)$ & \\
\hline Separated/divorced & $9(4.6)$ & $69(3.0)$ & $15(3.8)$ & \\
\hline \multicolumn{5}{|l|}{ Religion } \\
\hline Christian & $123(62.4)$ & $90(45.7)$ & $213(54.1)$ & \multirow{3}{*}{$0.001^{\star}$} \\
\hline Muslim & $74(37.6)$ & $103(52.3)$ & $177(44.9)$ & \\
\hline others & $0(0.0)$ & $4(2.0)$ & $4(1.0)$ & \\
\hline
\end{tabular}

Continuous variable i.e. mean age is presented as means \pm standard deviations and others are categorical variables presented as frequency counts and (percentages). Percentages are column percentages. $\mathrm{p}$ value is based on chi square test for categorical variable and independent sample T-test for continuous variable. ${ }^{*} \mathrm{p}$ value $<0.05$ shows a statistically significant relationship. 
Table 2. Clinical characteristics of screened participants.

\begin{tabular}{ccccc}
\hline $\begin{array}{c}\text { Clinical } \\
\text { characteristics }\end{array}$ & $\begin{array}{c}\text { Glaucoma } \\
\text { participants }\end{array}$ & $\begin{array}{c}\text { Cataract } \\
\text { participants }\end{array}$ & $\begin{array}{c}\text { All } \\
\text { participants }\end{array}$ & p value \\
Visual status & & & & \\
Normal & $79(40.1)$ & $71(36.0)$ & $150(38.1)$ & \\
Mild visual impairment & $16(8.1)$ & $69(35.0)$ & $85(21.6)$ & $<0.001$ \\
Moderate Visual impairment & $8(4.1)$ & $35(17.8)$ & $43(10.9)$ & \\
Severe Visual impairment & $4(2.0)$ & $6(3.0)$ & $10(2.5)$ & \\
Blind & $90(45.7)$ & $16(8.1)$ & $106(26.9)$ & \\
\hline
\end{tabular}

Medical therapy for glaucoma

$\begin{array}{cc}\text { Yes } & 185(94.4) \\ \text { no } & 11(5.6)\end{array}$

\begin{tabular}{|c|c|}
\hline \multicolumn{2}{|c|}{ Glaucoma participants using } \\
\hline B blockers for me & \\
\hline Yes & $167(84.8)$ \\
\hline no & $30(15.2)$ \\
\hline
\end{tabular}

Surgical therapy for glaucoma

$\begin{array}{lc}\text { Yes } & 57(28.9) \\ \text { no } & 140(71.1)\end{array}$

Family history of glaucoma

$\begin{array}{lc}\text { Yes } & 39(19.8) \\ \text { no } & 158(80.2)\end{array}$

Severity of glaucoma

$\begin{array}{cc}\text { Mild } & 18(9.1) \\ \text { Moderate } & 25(12.7) \\ \text { severe } & 154(78.2)\end{array}$

These categorical variables are presented as frequency counts and (percentages). Percentages are column percentages. $p$ value is based on chi square test. ${ }^{*} p$ value $<0.05$ showing statistically significant relationship.

Table 3. Prevalence of Anxiety and Depression amongst the Screened Participants.

\begin{tabular}{ccccc}
\hline & $\begin{array}{c}\text { Glaucoma } \\
\text { participants }\end{array}$ & $\begin{array}{c}\text { Cataract } \\
\text { participants }\end{array}$ & $\begin{array}{c}\text { All } \\
\text { participants }\end{array}$ & p value \\
\hline Anxiety & $70(35.5)$ & $43(21.8)$ & $113(28.7)$ & 0.001 \\
Depression & $48(24.4)$ & $7(3.6)$ & $55(14.0)$ & $<0.001$ \\
\hline
\end{tabular}

These categorical variables are presented as frequency counts and (percentages). Percentages are column percentages. $\mathrm{p}$ value is based on chi square test. ${ }^{*} \mathrm{p}$ value $<0.05$ showing statistically significant relationship. 


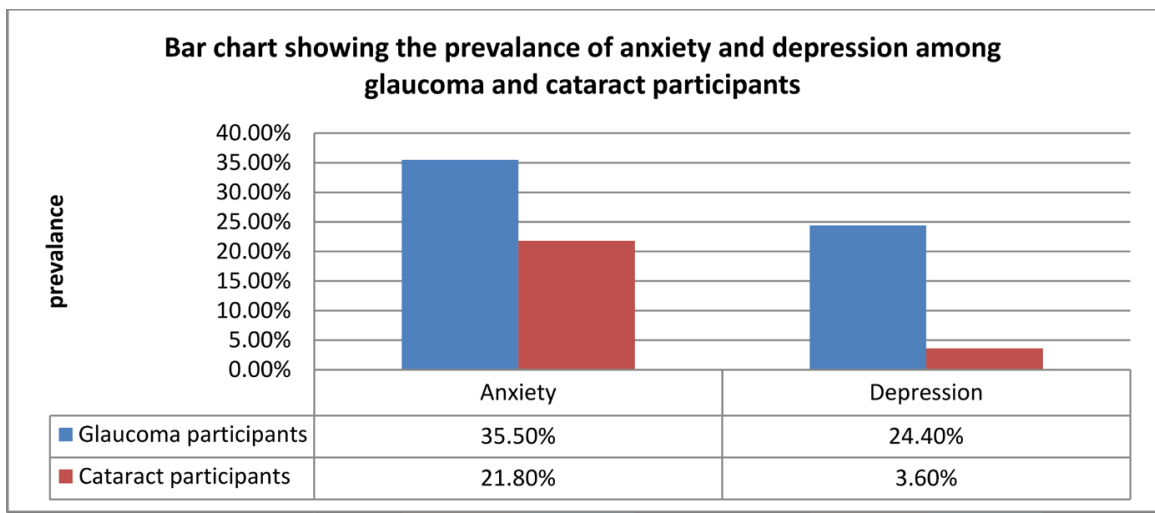

Figure 1. Bar chart showing the prevalence of anxiety and depression among glaucoma and cataract participants.

Table 4. Classification of anxiety and depression amongst the screened participants.

\begin{tabular}{ccccc}
\hline & $\begin{array}{c}\text { Glaucoma } \\
\text { participants }\end{array}$ & $\begin{array}{c}\text { Cataract } \\
\text { participants }\end{array}$ & $\begin{array}{c}\text { All } \\
\text { participants }\end{array}$ & p value \\
\hline $\begin{array}{c}\text { Anxiety } \\
\text { Mild }\end{array}$ & $31(15.7)$ & $29(14.7)$ & $60(15.2)$ & $0.001^{*}$ \\
Moderate & $29(14.7)$ & $13(6.6)$ & $42(10.7)$ & \\
severe & $10(5.1)$ & $1(0.5)$ & $11(2.8)$ & \\
Depression & & & & \\
Mild & $25(12.7)$ & $7(3.6)$ & $32(8.1)$ & $<0.001^{\star}$ \\
Moderate & $11(5.6)$ & $0(0.0)$ & $11(2.8)$ & \\
severe & $12(6.1)$ & $0(0.0)$ & $12(3.0)$ & \\
\hline
\end{tabular}

These categorical variables are presented as frequency counts and (percentages). Percentages are column percentages. $p$ value is based on chi square test. ${ }^{*} p$ value $<0.05$ showing statistically significant relationship.

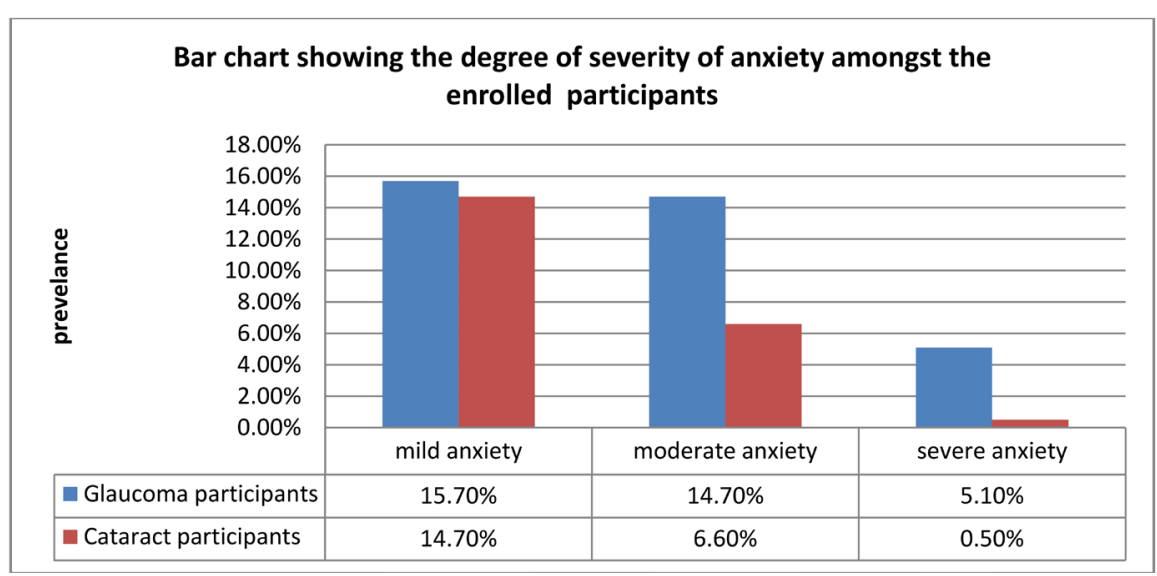

Figure 2. Bar chart showing the degree of severity of anxiety amongst the enrolled participants.

There was a statistically significant difference in the mean anxiety score and mean depression score comparing glaucoma and cataract patients (Table 5). 


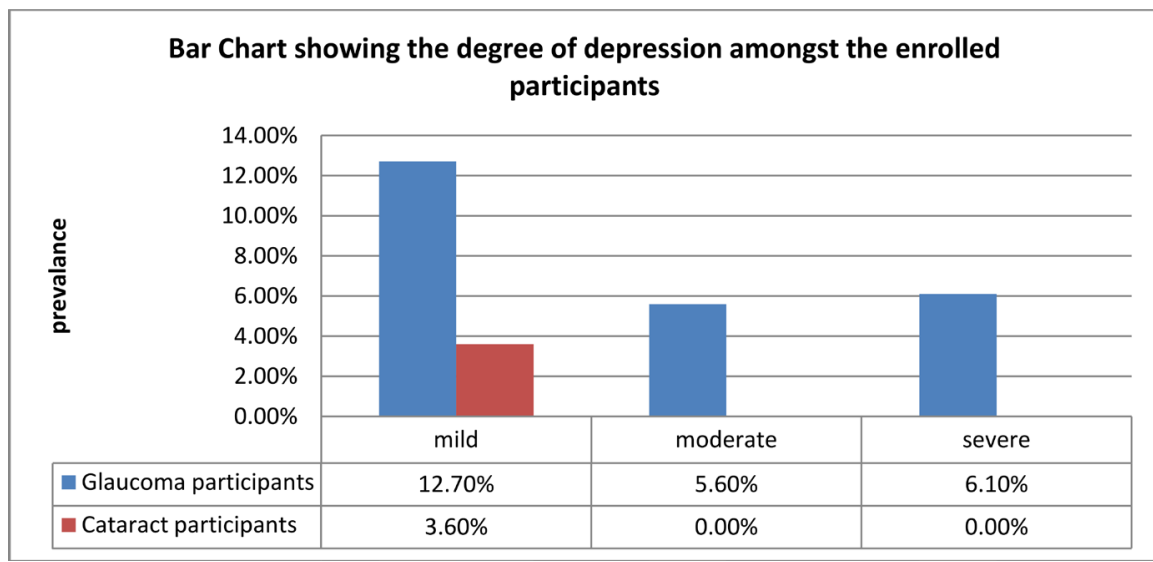

Figure 3. Bar chart showing the degree of depression amongst enrolled participants.

Table 5. Mean anxiety and depression score amongst screened participants.

\begin{tabular}{cccc}
\hline & Glaucoma participants & Cataract participants & p value \\
\hline Anxiety score & $6.21 \pm 4.74$ & $4.70 \pm 3.68$ & $<0.001$ \\
Depression score & $5.60 \pm 4.66$ & $3.05 \pm 2.46$ & $<0.001$ \\
\hline
\end{tabular}

The mean anxiety and depression score and are presented as means \pm standard deviations. $p$ value is based on independent sample T-test. ${ }^{*}$ p value $<0.05$ showing statistically significant relationship.

Risk factors among glaucoma participants found to be associated with anxiety were age and the severity of the disease. Glaucoma participants younger than 60 had a 2.7 times higher risk than those older than 60 to have anxiety (OR 2.7, 95\% CI: $1.3-6.3 \mathrm{p}=0.009)$. Those with severe glaucoma were 9.6 times more likely than those with mild glaucoma to be anxious (OR 9.6, 95\% CI: $1.7-54.44 \mathrm{p}=$ 0.011) (Table 6).

The risk factors found to have a significant association with anxiety amongst cataract participants were only sociodermographic factors. These factors are employment and marital status. Those employed were 6.4 times more likely than those not employed to have anxiety (OR 6.4, 95\% CI: $2.1-20.0 \mathrm{p}=$ $0.001)$. Those separated or divorced were 10.3 times more likely to be anxious than those married (OR 10.3, 95\% CI: $1.4-78.4 \mathrm{p}=0.024)$. Visual status was not found to be a significant risk factor for anxiety amongst cataract participants (Table 7).

Overall, among all the participants the risk factors significantly associated with anxiety include visual status, sex and marital status. Blind participants were 2.4 times more likely to be anxious than those not visually impaired (OR 2.4, 95\% CI: $1.3-4.4 \mathrm{p}=0.007)$. Females were 1.8 times as likely as males to be anxious (OR 1.8, 95\% CI: $1.1-3.0 \mathrm{p}=0.018$ ). Those separated or divorced were 3.2 times more likely to be anxious than those married (OR 3.2, 95\% CI: $1.1-10.1 \mathrm{p}=$ 0.045) (Table 8).

Severity of disease, age and employment status were the risk factors found to have a significant relationship with depression among glaucoma participants. Those with severe glaucoma were 9.6 times more likely to have depression as 
Table 6. Logistic regression of anxiety among glaucoma participants by sociodemographic and clinical characteristics.

\begin{tabular}{|c|c|c|}
\hline Sociodemographic and clinical characteristics & $\begin{array}{l}\text { Univariate Logistic Regression } \\
\text { OR, }(95 \% \mathrm{CI}), \mathrm{p}\end{array}$ & $\begin{array}{c}\text { Multivariate Logistic Regression } \\
\text { OR, }(95 \% \mathrm{CI}), \mathrm{p}\end{array}$ \\
\hline \multicolumn{3}{|l|}{ Age } \\
\hline$>60$ & 1 & 1 \\
\hline$<60$ & $1.671,(0.928-3.009), p=0.087$ & $2.922,(1.306-6.536), p=0.009^{*}$ \\
\hline \multicolumn{3}{|l|}{ Sex } \\
\hline Male & 1 & 1 \\
\hline Female & $1.563,(0.865-2.825), \mathrm{p}=0.139$ & $2.003,(0.964-4.183), p=0.063$ \\
\hline \multicolumn{3}{|l|}{ Education } \\
\hline Tertiary & 1 & 1 \\
\hline Nil & $1.310,(0.485-3.539), p=0.595$ & $1.101,(0.320-3.793), \mathrm{p}=0.879$ \\
\hline Primary & $0.895,(0.412-1.945), p=0.780$ & $1.022,(0.390-2.677), \mathrm{p}=0.965$ \\
\hline Secondary & $1.120,(0.513-2.448), p=0.776$ & $1.213,(0.467-3.152), \mathrm{p}=0.692$ \\
\hline \multicolumn{3}{|l|}{ Employment } \\
\hline Others(unemployed/retired and students) & 1 & 1 \\
\hline Employed/selfemployed & $1.081,(0.603-1.935), \mathrm{p}=0.794$ & $0.718,(0.339-1.523), \mathrm{p}=0.388$ \\
\hline \multicolumn{3}{|l|}{ Marital status } \\
\hline Married & 1 & 1 \\
\hline Single & $0.742,(0.188-2.932), \mathrm{p}=0.670$ & $0.571,(0.098-3.316), \mathrm{p}=0.532$ \\
\hline Widowed & $1.424,(0.704-2.879), \mathrm{p}=0.325$ & $1.100,(0.469-2.584), \mathrm{p}=0.826$ \\
\hline Separated/divorced & $2.472,(0.633-9.659), p=0.193$ & $1.336,(0.266-6.698), p=0.725$ \\
\hline \multicolumn{3}{|l|}{ Religion } \\
\hline Muslim & 1 & 1 \\
\hline Christian & $1.565,(0.846-2.896), \mathrm{p}=0.154$ & $1.329,(0.653-2.706), p=0.433$ \\
\hline \multicolumn{3}{|l|}{ Visual status } \\
\hline Normal & 1 & 1 \\
\hline Mild & $0.432,(0.089-2.087), p=0.296$ & $0.171,(0.028-1.027), \mathrm{p}=0.053$ \\
\hline Moderate & $1.295,(0.298-5.638), p=5.638$ & $1.031,(0.194-5.490), \mathrm{p}=0.971$ \\
\hline Severe & $0.864,(0.085-8.783), p=0.901$ & $0.298,(0.023-6.698), p=0.354$ \\
\hline blind & $2.321,(1.222-4.408), \mathrm{p}=0.010^{\star}$ & $1.493,(0.635-3.511), p=0.358$ \\
\hline \multicolumn{3}{|l|}{ Severity group } \\
\hline Mild & 1 & 1 \\
\hline Moderate & $1.524,(0.247-9.383), \mathrm{p}=0.650$ & $2.679,(0.384-18.661), p=0.320$ \\
\hline severe & $5.843,(1.298-26.299), \mathrm{p}=0.021^{\star}$ & $9.588,(1.687-54.444), \mathrm{p}=0.011^{\star}$ \\
\hline \multicolumn{3}{|l|}{ Medical therapy } \\
\hline No & 1 & 1 \\
\hline yes & $2.654,(0.577-12.639), p=0.220$ & $4.918,(0.563-42.943), p=0.150$ \\
\hline \multicolumn{3}{|l|}{ B blockers } \\
\hline $\begin{array}{l}\text { No } \\
\text { yes }\end{array}$ & $1.151,(0.506-2.618), p=0.737$ & $0.593,(0.171-2.055), \mathrm{p}=0.410$ \\
\hline \multicolumn{3}{|l|}{ Surgical therapy } \\
\hline No & 1 & 1 \\
\hline yes & $0.677,(0.349-1.312), p=0.677$ & $0.734,(0.336-1.064), p=0.438$ \\
\hline \multicolumn{3}{|l|}{ Family history } \\
\hline No & 1 & 1 \\
\hline yes & $1.698,(0.834-3.451), \mathrm{p}=0.144$ & $2.013,(0.832-4.871), \mathrm{p}=0.121$ \\
\hline
\end{tabular}

${ }^{*} \mathrm{P}$ value $<0.05$ showing statistically significant relationship, $\mathrm{OR}=$ Odd's Ratio; $\mathrm{CI}=$ Confidence Interval; $\mathrm{p}=$ Level of significance. 
Table 7. Logistic regression of anxiety among cataract participants by sociodemographic and clinical characteristics.

\begin{tabular}{|c|c|c|}
\hline Sociodemographic and clinical characteristics & $\begin{array}{l}\text { Univariate Logistic Regression OR, } \\
\text { (95\% CI), p }\end{array}$ & $\begin{array}{c}\text { Multivariate Logistic Regression } \\
\text { OR, }(95 \% \mathrm{CI}), \mathrm{p}\end{array}$ \\
\hline \multicolumn{3}{|l|}{ Age } \\
\hline$>60$ & 1 & 1 \\
\hline$<60$ & $1.657(0.835-3.288) \mathrm{p}=0.149$ & $0.590(0.185-1.879) \mathrm{p}=0.372$ \\
\hline \multicolumn{3}{|l|}{ Sex } \\
\hline Male & 1 & 1 \\
\hline female & $2.256(1.136-4.480) \mathrm{p}=0.020^{*}$ & $2.379(0.975-5.805) \mathrm{p}=0.057$ \\
\hline \multicolumn{3}{|l|}{ Education } \\
\hline Tertiary & 1 & 1 \\
\hline Nil & $0.599(0.221-1.621) \mathrm{p}=0.313$ & $0.262(0.064-1.074) \mathrm{p}=0.063$ \\
\hline Primary & $0.365(0.144-0.927) \mathrm{p}=0.034^{*}$ & $0.301(0.081-1.123) \mathrm{p}=0.074$ \\
\hline Secondary & $0.479(0.174-1.316) \mathrm{p}=0.153$ & $0.240(0.065-0.890) \mathrm{p}=0.033^{*}$ \\
\hline \multicolumn{3}{|l|}{ Employment } \\
\hline Others (unemployed/retired and students) & 1 & 1 \\
\hline Employed/self employed & $2.686(1.205-5.988) \mathrm{p}=0.016^{*}$ & $6.404(2.051-19.994) \mathrm{p}=0.001^{*}$ \\
\hline \multicolumn{3}{|l|}{ Marital status } \\
\hline Married & 1 & 1 \\
\hline Single & $3.733(0.505-27.612) \mathrm{p}=0.197$ & $10.353(0.835-128.419) \mathrm{p}=0.069$ \\
\hline Widowed & $0.688(0.279-1.694) p=0.416$ & $0.400(0.137-1.164) \mathrm{p}=0.093$ \\
\hline Separated/divorced & $7.467(1.305-42.734) p=0.024^{*}$ & $10.335(1.363-78.354) \mathrm{p}=0.024^{*}$ \\
\hline \multicolumn{3}{|l|}{ Visual status } \\
\hline Normal & 1 & 1 \\
\hline Mild & $0.859(0.398-1.8530 \mathrm{p}=0.698$ & $1.288(0.379-4.384) \mathrm{p}=0.685$ \\
\hline Moderate & $0.332(0.104-1.062) \mathrm{p}=0.063$ & $0.480(0.105-2.193) \mathrm{p}=0.343$ \\
\hline Severe & $1.368(0.231-8.089) \mathrm{p}=0.729$ & $6.171(0.709-53.715) p=0.099$ \\
\hline blind & $0.391(0.081-1.883) \mathrm{p}=0.242$ & $0.465(0.065-3.347) \mathrm{p}=0.447$ \\
\hline
\end{tabular}

${ }^{\star} \mathrm{P}$ value $<0.05$ showing statistically significant relationship, $\mathrm{OR}=$ Odd's Ratio; $\mathrm{CI}=$ Confidence Interval; $\mathrm{p}=$ Level of significance.

Table 8. Logistic regression of anxiety among all participants by sociodemographic and clinical characteristics.

\begin{tabular}{|c|c|c|}
\hline Sociodemographic and clinical characteristics & $\begin{array}{l}\text { Univariate Logistic Regression OR, } \\
(95 \% \mathrm{CI}), \mathrm{p}\end{array}$ & $\begin{array}{c}\text { Multivariate Logistic Regression } \\
\text { OR, }(95 \% \mathrm{CI}), \mathrm{p}\end{array}$ \\
\hline Cataract participants & 1 & 1 \\
\hline Glaucoma participants & $2.018(1.292-3.152) \mathrm{p}=0.002^{\star}$ & $1.409(0.820-2.421) \mathrm{p}=0.214$ \\
\hline \multicolumn{3}{|l|}{ Age } \\
\hline$>60$ & 1 & 1 \\
\hline$<60$ & $1.711(1.011-2.659) \mathrm{p}=0.017^{\star}$ & $1.504(0.855-2.646) \mathrm{p}=0.156$ \\
\hline \multicolumn{3}{|l|}{ Sex } \\
\hline Male & 1 & 1 \\
\hline female & $1.822(1.171-2.834) \mathrm{p}=0.008^{*}$ & $1.829(1.110-3.012) \mathrm{p}=0.018^{*}$ \\
\hline
\end{tabular}




\section{Continued}

\begin{tabular}{|c|c|c|}
\hline \multicolumn{3}{|l|}{ Education } \\
\hline Tertiary & 1 & 1 \\
\hline Nil & $0.812(0.408-1.614) \mathrm{p}=0.552$ & $0.830(0.362-1.903) \mathrm{p}=0.659$ \\
\hline Primary & $0.581(0.322-1.049) \mathrm{p}=0.072$ & $0.668(0.341-1.311) \mathrm{p}=0.241$ \\
\hline Secondary & $0.804(0.436-1.481) \mathrm{p}=0.484$ & $0.792(0.406-1.544) \mathrm{p}=0.493$ \\
\hline \multicolumn{3}{|l|}{ Employment } \\
\hline Others(unemployed/ retired and students) & 1 & 1 \\
\hline Employed/self employed & $1.382(0.884-2.163) \mathrm{p}=0.156$ & $1.462(0.855-2.501) p=0.165$ \\
\hline \multicolumn{3}{|l|}{ Marital status } \\
\hline Married & 1 & 1 \\
\hline Single & $1.340(0.443-4.049) \mathrm{p}=0.604$ & $1.144(0.330-3.963) \mathrm{p}=0.832$ \\
\hline Widowed & $1.063(0.624-1.814) \mathrm{p}=0.821$ & $0.964(0.525-1.773) \mathrm{p}=0.907$ \\
\hline Separated/divorced & $4.020(1.384-11.679) \mathrm{p}=0.011^{*}$ & $3.214(1.024-10.082) \mathrm{p}=0.045^{*}$ \\
\hline \multicolumn{3}{|l|}{ Visual status } \\
\hline Normal & 1 & 1 \\
\hline Mild & $0.760(0.402-1.433) \mathrm{p}=0.396$ & $1.008(0.489-2.075) \mathrm{p}=0.984$ \\
\hline Moderate & $0.477(0.198-1.152) \mathrm{p}=0.100$ & $0.722(0.275-1.892) \mathrm{p}=0.507$ \\
\hline Severe & $1.139(0.281-4.617) \mathrm{p}=0.855$ & $1.622(0.369-7.126) \mathrm{p}=0.522$ \\
\hline blind & $1.930(1.141-3.264) \mathrm{p}=0.014^{\star}$ & $2.358(1.258-4.418) \mathrm{p}=0.007^{\star}$ \\
\hline
\end{tabular}

${ }^{*} \mathrm{P}$ value $<0.05$ showing statistically significant relationship, $\mathrm{OR}=$ Odd's Ratio; $\mathrm{CI}=\mathrm{Confidence} \mathrm{Interval;} \mathrm{p}=\mathrm{Level}$ of significance.

compared to those with mild disease (OR 9.6, 95\% CI: $1.7-54.4 \mathrm{p}=0.011$ ). Those younger than sixty were 2.9 times more likely to have depression than those older than sixty (OR 2.9, 95\% CI: $1.3-6.5 \mathrm{p}=0.009$ ). Those not in active employment were two times more depressed than those in active employment (OR 2.0, 95\% CI: 1.0 - $7.2 \mathrm{p}=0.042$ ). Glaucoma participants on B blockers were 5.3 times more likely to be depressed than those not on B Blockers (OR 5.3, 95\% CI: $1.2-23.2 p=0.026$ ) but this was no longer statistically significant when sociodermographic and clinical variables were controlled for (OR 3.6, 95\% CI: 0.5 $25.8 \mathrm{p}=0.205)$ (Table 9).

Amongst all the participants, glaucoma patients had a 3.9 times higher risk than cataract patients to be depressed (OR 3.9, 95\% CI: $1.5-10.8 \mathrm{p}=0.007$ ). The risk factors found to be associated with depression among all the participants include visual status, age, education and employment. Blind participants were 7.1 times more likely to have depression (OR 7.1, 95\% CI: $2.8-17.7 \mathrm{p}<0.001$ ). Those younger than sixty were 2.6 times more likely to be depressed than those older than sixty (OR 2.6, 95\% CI: $1.1-6.1 \mathrm{p}=0.026$ ). Those with no formal education (OR7.5, 95\% CI: $2.0-29.2 \mathrm{p}=0.003)$, those with at least primary education (OR 3.4, 95\% CI: $1.0-11.5 \mathrm{p}=0.048$ ) and those with at least secondary education (OR 6.7, 95\% CI $2.1-21.8 \mathrm{p}=0.002)$ were found to be more depressed than those with tertiary education. Those unemployed were 2.8 times more likely to be depressed than those employed (OR 2.8, 95\% CI: $1.3-6.2 \mathrm{p}=0.009$ ) (Table 10). 
Table 9. Logistic regression of depression among glaucoma participants by sociodemographic and clinical characteristics.

\begin{tabular}{|c|c|c|}
\hline Sociodemographic and clinical characteristics & $\begin{array}{l}\text { Univariate Logistic Regression OR, } \\
\qquad(95 \% \mathrm{CI}), \mathrm{p}\end{array}$ & $\begin{array}{c}\text { Multivariate Logistic Regression } \\
\text { OR, (95\% CI), p }\end{array}$ \\
\hline \multicolumn{3}{|l|}{ Age } \\
\hline$>60$ & 1 & 1 \\
\hline$<60$ & $1.187(0.617-2.285) \mathrm{p}=0.607$ & $4.729(1.654-13.518) \mathrm{p}=0.004^{*}$ \\
\hline \multicolumn{3}{|l|}{ Sex } \\
\hline Male & 1 & 1 \\
\hline female & $0.620(0.311-1.239) \mathrm{p}=0.176$ & $1.152(0.448-2.964) p=0.769$ \\
\hline \multicolumn{3}{|l|}{ Education } \\
\hline Tertiary & 1 & 1 \\
\hline Nil & $3.188(1.058-13.562) \mathrm{p}=0.041^{*}$ & $4.749(0.993-22.726) p=0.051$ \\
\hline Primary & $2.816(0.948-8.370) \mathrm{p}=0.062$ & $6.695(1.576-28.441) \mathrm{p}=0.010^{*}$ \\
\hline Secondary & $5.222(1.796-15.178) \mathrm{p}=0.002^{*}$ & $7.964(2.119-29.930) p=0.002^{*}$ \\
\hline \multicolumn{3}{|l|}{ Employment } \\
\hline Employed/self employed & 1 & 1 \\
\hline Others (unemployed/retired and students) & $1.408(0.733-2.704) \mathrm{p}=0.305$ & $2.027(1.035-7.181) \mathrm{p}=0.042^{*}$ \\
\hline \multicolumn{3}{|l|}{ Marital status } \\
\hline Married & 1 & 1 \\
\hline Single & $0.272(0.034-2.203 p=0.223$ & $0.140(0.011-1.756) \mathrm{p}=0.128$ \\
\hline Widowed & $0.721(0.315-1.649) \mathrm{p}=0.438$ & $0.832(0.271-2.555) \mathrm{p}=0.747$ \\
\hline Separated/divorced & $0.778(0.154-3.919) \mathrm{p}=0.761$ & $0.293(0.035-2.432) \mathrm{p}=0.256$ \\
\hline \multicolumn{3}{|l|}{ Visual status } \\
\hline Normal & 1 & 1 \\
\hline Severe & $3.429(0.313-37.512) \mathrm{p}=0.313$ & $3.454(0.230-51.919) \mathrm{p}=0.370$ \\
\hline blind & $8.067(3.348-19.439) \mathrm{p}<0.001^{*}$ & $10.821(2.764-42.356) \mathrm{p}=0.001^{*}$ \\
\hline \multicolumn{3}{|l|}{ Severity group } \\
\hline Mild & 1 & 1 \\
\hline Moderate & $0.696(0.089-5.465) p=0.730$ & $0.944(0.090-9.915) \mathrm{p}=0.962$ \\
\hline severe & $3.2(0.706-14.5) \mathrm{p}=0.131$ & $0.738(0.095-5.748) \mathrm{p}=0.776$ \\
\hline \multicolumn{3}{|l|}{ B blockers } \\
\hline No & 1 & 1 \\
\hline yes & $5.322(1.219-23.245) \mathrm{p}=0.026^{\star}$ & $3.583(0.497-25.806) p=0.205$ \\
\hline \multicolumn{3}{|l|}{ Surgical therapy } \\
\hline No & 1 & 1 \\
\hline yes & $1.494(0.747-2.990) \mathrm{p}=0.256$ & $3.663(1.352-9.928) \mathrm{p}=0.011^{\star}$ \\
\hline \multicolumn{3}{|l|}{ Family history } \\
\hline No & 1 & 1 \\
\hline yes & $0.915(0.400-2.095) \mathrm{p}=0.834$ & $1.896(0.599-5.997) \mathrm{p}=0.276$ \\
\hline
\end{tabular}

${ }^{\star} \mathrm{P}$ value $<0.05$ showing statistically significant relationship, $\mathrm{OR}=$ Odd's Ratio; $\mathrm{CI}=$ Confidence Interval; $\mathrm{p}=$ Level of significance. 
Table 10. Logistic regression of depression among all participants by sociodemographic and clinical characteristics.

\begin{tabular}{|c|c|c|}
\hline Sociodemographic and clinical characteristics & $\begin{array}{l}\text { Univariate Logistic Regression OR, } \\
\qquad(95 \% \mathrm{CI}), \mathrm{p}\end{array}$ & $\begin{array}{c}\text { Multivariate Logistic Regression } \\
\text { OR, }(95 \% \mathrm{CI}), \mathrm{p}\end{array}$ \\
\hline Cataract participants & 1 & 1 \\
\hline Glaucoma participants & $8.744(3.845-19.882) \mathrm{p}<0.001^{*}$ & $3.973(1.461-10.808) p=0.007^{*}$ \\
\hline \multicolumn{3}{|l|}{ Age } \\
\hline$>60$ & 1 & 1 \\
\hline$<60$ & $1.100(0.617-1.961) \mathrm{p}=0.743$ & $2.608(1.120-6.071) \mathrm{p}=0.026^{*}$ \\
\hline \multicolumn{3}{|l|}{ Sex } \\
\hline Male & 1 & 1 \\
\hline female & $0.969(0.539-1.741) \mathrm{p}=0.915$ & $1.259(0.585-2.710) \mathrm{p}=0.556$ \\
\hline \multicolumn{3}{|l|}{ Education } \\
\hline Tertiary & 1 & 1 \\
\hline Nil & $3.6(1.2-10.8) \mathrm{p}=0.022^{*}$ & $7.565(1.960-29.206) p=0.003^{*}$ \\
\hline Primary & $1.96(0.686-5.601) p=0.209$ & $3.412(1.008-11.542) \mathrm{p}=0.048^{*}$ \\
\hline Secondary & $4.657(1.687-12.855) \mathrm{p}=0.003^{*}$ & $6.708(2.067-21.764) p=0.002^{*}$ \\
\hline \multicolumn{3}{|l|}{ Employment } \\
\hline Employed/self employed & 1 & 1 \\
\hline Others(unemployed/ retired and students) & $2.102(1.179-3.748) \mathrm{p}=0.012^{*}$ & $2.847(1.302-6.223) \mathrm{p}=0.009^{\star}$ \\
\hline \multicolumn{3}{|l|}{ Marital status } \\
\hline Married & 1 & 1 \\
\hline Single & $0.434(0.055-3.395) \mathrm{p}=0.426$ & $0.118(0.011-1.288) \mathrm{p}=0.080$ \\
\hline Widowed & $0.868(0.424-1.778) \mathrm{p}=0.6 .99$ & $0.853(0.335-2.169) \mathrm{p}=0.738$ \\
\hline Separated/divorced & $2.210(0.670-7.289) \mathrm{p}=0.193$ & $1.556(0.315-7.685) \mathrm{p}=0.587$ \\
\hline \multicolumn{3}{|l|}{ Visual status } \\
\hline Normal & 1 & 1 \\
\hline Mild & $0.922(0.269-3.161) p=0.897$ & $1.228(0.319-4.727) \mathrm{p}=0.765$ \\
\hline Moderate & & 0.000 \\
\hline Severe & $7.607(1.650-35.081) \mathrm{p}=0.009^{*}$ & $8.825(1.648-47.269) \mathrm{p}=0.011^{*}$ \\
\hline blind & $10.597(4.701-23.887) \mathrm{p}<0.001^{*}$ & $7.084(2.838-17.679) \mathrm{p} \leq 0.001^{*}$ \\
\hline
\end{tabular}

${ }^{*} \mathrm{P}$ value $<0.05$ showing statistically significant relationship, $\mathrm{OR}=$ Odd's Ratio; $\mathrm{CI}=$ Confidence Interval; $\mathrm{p}=$ Level of significance.

\section{Discussion}

Majority of the glaucoma participants were elderly with a mean age of 62 years. They were much older than participants in other similar studies in urban areas within the country such as glaucoma clinic patients in University of Benin, Teaching Hospital (UBTH) had a mean age of 57 years [5], 59 years amongst patients seen in Lagos University Teaching Hospital (LUTH) [7] and 55 years among patients attending two ophthalmic centers in Abuja [8]. It was also higher than similar studies abroad such as seen amongst patients in Karachi who had a mean age of 56.1 years [24] and in various centers in the US where the mean age was 57.5 years [25]. This is probably due to the fact that the study was in a rural area 
and it is presumed that rural areas have more elderly population than in urban areas. Most of the patients were also males which is in keeping with other studies within the country [5] [7] [8] as males have been found to have better access to health care services. The employment status also differed within this rural area as compared to the other studies in urban areas. Those employed were more than patients seen attending LUTH [7] and UBTH [5] where $46 \%$ of the glaucoma clinic patients were employed as compared to $52 \%$ in this study even though majority of the participants in this study were above 60 than in the other studies where majority were much younger. Very few were unemployed (2.5\%) as compared to $18 \%$ in Lagos [7], 12\% in Benin [5] and 13\% in Abuja [8]. This could be due to the higher cost of living in urban regions and challenges with job seeking in urban areas than in this rural area where majority were self employed. The retirees $(42 \%)$ in this study were also higher than other studies in urban areas such as $38 \%$ in Benin [5], 36\% in Lagos [7], 25\% in Abuja [8]. This is probably because of the more elderly population in rural areas and most retirees usually relocate to their villages which are usually in rural areas. The educational status was much lower as only $26 \%$ of glaucoma participants had tertiary education as compared to 54\% in Lagos [7], although it was higher than those seen in Benin [5] where only $12 \%$ had a tertiary education. Also $12 \%$ did not have a formal education which also differed from the 5\% in Lagos [7] and 18\% in Benin [5]. It was also noted that there was no statistically significant difference between the age and sex between the glaucoma and cataract participants. Although there were differences in their educational status as those that had tertiary education were more amongst the glaucoma participants than the those with cataracts and those without any formal education were more amongst cataract participants. This difference could be due to the fact that the glaucoma participants have been known to move around looking for solutions. Despite this differences in educational status the cataract participants were found to be more employed than those with glaucoma as more of the glaucoma participants were retired and this could be due to challenges the glaucoma participants have with their vision as over three quarter had severe glaucoma and almost half were blind. There were also differences in the clinical characteristics between both groups as almost half of the glaucoma participants were blind as compared to $8.1 \%$ of those with cataracts, even though more of the glaucoma participants had no visual impairment. This is because glaucoma is a chronic irreversible disease unlike the cataract patients where vision can be restored through surgery so glaucoma patients that will be blind will generally be more than cataract patients seen within a period of time. Although these differences exist they were addressed during the analysis with a logistic regression which took care of confounders in other to ascertain the odds of anxiety and depression between the two groups and the independent risk factors associated with these psychological disorders.

The clinical characteristics of the participants also differed from other studies. In this study almost half of the glaucoma participants were blind and over a 
third had severe glaucoma. This is much higher than patients seen in UBTH [5], LUTH [7], Abuja [8] were half of the glaucoma subjects had severe disease and also higher than glaucoma subjects in other studies abroad [3] [4] [12].

Over a third of glaucoma participants were found to be anxious which shows that anxiety is an issue that needs to be addressed among these patients. There is a need for appropriate and adequate counseling. This was similar to findings seen in patients in Karachi [24] were 33\% of the patients were anxious. Although a much lower value of $10 \%$ was seen amongst patients in UBTH [5]. This could be due to small sample size of 50 participants although only eleven of these were accessed for anxiety. Lower prevalence was seen in clinic patients in Shanghai, China (22.92\%) [12], Turkey (14\%) [11] and Japan (13\%) [26] although these three centers were teaching hospitals and were located in developed countries, so it can be presumed that patients seen in teaching hospital are less anxious than those seen within this community eye hospital within a rural area. Also the challenges in developing countries could account for the higher prevalence gotten in studies done in Lagos [7] and Karachi [24].

Despite the fact that $35.5 \%$ of cases as compared to $22 \%$ of cataract participants were anxious, there was no statistically significant difference in anxiety between the two groups on logistic regression after controlling for sociodermographic and clinical factors. Hence glaucoma patients are not more anxious than cataract patients attending this center. Anxiety was also found to be higher among glaucoma patients as compared to controls seen amongst clinic patients in Japan [26] as reported by Mabuchi where $10.9 \%$ of cases were anxious as compared to $5.2 \%$ of controls which were also cataract patients.

The study also showed that those younger than 60 were 2.9 times more likely to be anxious than those older than 60 . This is probably because this age group is still active in their places of employment. The fear of losing their vision which will affect their productivity at work and their roles in the family as compared to those older than 60 who are more likely to be retired. Similar findings were reported by Dawodu et al. amongst glaucoma clinic patients seen in UBTH [5], Zhou et al. amongst patients seen in Shaghai, China [12] and by Mabuchi et al. in Japan [27].

The severity of the disease was also a risk factor for anxiety. This is expected as the risk of blindness is higher in those with severe disease so fear of the disease affecting their quality of life.

The risk factors for anxiety differed amongst cataract and glaucoma participants as those for the controls were mainly sociodermographic factor such as employment and marital status. Among all the participants females had two times higher risk of being anxious. Similar reports were reported by participants in Ankara,Turkey [11] and Shanghai, China [12].

Despite the difference in sociodermographic and clinical factors between the patients in this study and those in the other studies the prevalence of depression was similar, as $24.4 \%$ of the glaucoma participants had depression as compared 
to $22 \%$ of patients in LUTH [7] and $25.5 \%$ of patients in Abuja [8]. Although a much lower prevalence of $6 \%$ was gotten amongst patients seen in UBTH [5] and this could be because of the small sample size among patients in UBTH as there were 50 participants but only 11 were accessed for depression. Similar findings were noted amongst clinic patients in Israel (21.1\%) [3] but much lower prevalence amongst seen in California (7.4\%) [4] and $10.9 \%$ seen in patients in the US [28] and Japan [26]. These differences could arise because the higher prevalence's were seen in developing countries as compared to the others in developed countries where there is a presumed better standard of living.

There was a significant difference between the glaucoma and cataract participants as the glaucoma participants had a four times higher risk of having depression. Similar findings were reported amongst clinic patients in Abuja [8] even though the controls were those without visual impairment as compared tothis study where the comparative group are cataract patients with various ranges of visual status. This was also in keeping with studies amongst clinic patients in LUTH [7], Ankara Turkey [11] and Japan [26] and a population based study in the US [28] and differed from the studies done in Isreal [3], California [4] and in UBTH [5] were there was no difference with controls or the general population.

The independent risk factors for depression were the presence of the disease, younger age, lower educational status, blindness and those that had surgery. The younger age group is explainable because of the impact of the disease on their quality of life which will affect their role at their work place and families. A diverse picture was seen amongst patients seen in UBTH [5] were the elderly were more depressed. Educational status is a measure of economic status of these individuals and those with lower educational status being more depressed could also be as a result of the challenges of management of this chronic blinding disease. Similar findings were seen amongst patients attending UBTH [5] and a different picture was seen in Carlifornia [3] were those with college education were 4 times more depressed than those with high school education. This difference could have arisen because of the various pressures in the lifestyle of the different regions. Those that were unemployed were two times more likely to be depressed than those not employed. This could be as a result of the challenges with coping with the financial challenges in their day to day activities and also the challenges with glaucoma and its management. Similar findings were reported in clinic patients seen within cosmopolitican regions in Abuja [8] and Shanghai, China [12]. Patients that had surgery were four times more likely to be depressed. This could be due to the expectation of the patients following surgery hence the need for proper and adequate counseling.

The strengths of the study include the fact that it is a comparative crossectional study. A logistic regression was done in comparing anxiety and depression between cataract and glaucoma participants so we could actually determine the odds of having these psychological disorders and to identify independent risk 
factors. The limitations of the study were that the sampling was opportunistic so cannot be generalisable to the entire southwestern part of the country.

\section{Conclusion}

In conclusion Anxiety and Depression were found in $35.5 \%$ and $24.4 \%$ of glaucoma patients as compared to $21.8 \%$ and $3.6 \%$ of cataract patients respectively. Glaucoma patients were 4 times more likely to be depressed than patients with cataracts. These psychological issues occur in both glaucoma and cataract patients. There was no statistically significant difference in anxiety between the two groups after controlling for sociodermographic and clinical variables, although the presence of glaucoma was an independent risk factor for depression as glaucoma patients were four times more likely to be depressed than cataract patients. There is a need to address these psychological issues at the community level in other to improve the quality of life of these patients. It is also important to identify those at risk in other to curb the growing trend/concern.

\section{Recommendations}

1) To train some glaucoma patients as counselors for glaucoma patients within the center.

2) Advocate for the government to improve access to education and to create more job opportunities especially for the visually challenged.

3) There is a need for screening of glaucoma in other to identify early cases as over $3 / 4$ had severe glaucoma and almost $1 / 2$ were blind.

4) There is a need for training and equipping the community health workers and nurses with counseling and psychological assessment skills in other to identify those at risk and manage.

5) There is also a need for regular and occasional psychologist and psychiatrist visits to the patients through seminars and glaucoma support group meetings.

\section{Acknowledgements}

I want to acknowledge Dr. Kunle Hassan and all staff of the Deseret Community Vision Institute, Ijebu Mushin and Eye Foundation Hospital Group for their immense support and success towards the study.

\section{Conflicts of Interest}

The authors declare no conflicts of interest regarding the publication of this paper.

\section{References}

[1] Quigley, H.A. (1996) Number of People with Glaucoma Worldwide. British Journal of Ophthalmology, 80, 389-393. https://doi.org/10.1136/bjo.80.5.389

[2] Kyari, F., Gudlavalleb, M.V.S., Sivsubramaniam, S., Gilbert, C.E., Abdull, M.M., Eritekumme, G. and Foster, A. (2009) Prevalence of Blindness and Visual Impair- 
ment in Nigeria: The National Blindness and Visual Impairment Survey. Investigative Ophthalmology \& Visual Science, 50, 2033-2039.

https://doi.org/10.1167/iovs.08-3133

[3] Weiss, G., Yaakov, G., Bartov and Zvia, B. (2011) Compliance with Eye Care in Glaucoma Patients with Comorbid Depression. Israel Medical Association Journal, 13, 730-734.

[4] Wilson, M.R., Coleman, A.L., Yu, F., Fong, S.I., Bing, E.G. and Kim, M.H. (2002) Depression in Patients with Glaucoma as Measured by Self-Report Surveys. Ophthalmology, 109, 1018-1022. https://doi.org/10.1016/S0161-6420(02)00993-4

[5] Dawodu, O.A., Otakpo, A. and Ukponmwan, C. (2004) Common Psychiatric Disorders in Glaucoma Patients as Seen at the University of Benin Teaching Hospital, Benin City, Nigeria. Journal of Biomedical Sciences, 3, 42-47. https://doi.org/10.4314/jmbr.v3i1.10655

[6] Mitsonis, C., Dimopoulos, N., Psarrav, V., Santa, Z., Letsas, C. and Mitsonis, M. (2006) Depression and Anxiety in Patients with Glaucoma: A Prospective, Case Controls Study. Annals of General Psychiatry, 5, 10-20. https://doi.org/10.1186/1744-859X-5-S1-S316

[7] Akindipe, T.O., Aina, O.F. and Onakoya, A.O. (2011) Risk of Depression and Subjective Quality of Life among Attendees of a West African Glaucoma Clinic. International Journal of Medicine and Medical Sciences, 1, 31-34.

[8] Okudo, A.C. (2013) Visual Function and Quality of Life of Primary Open Angle Glaucoma Patient Attending Ophthalmic Centers in Abuja. Dissertation for the Award of Fellowship of National Post Graduate Medical College of Nigeria in Ophthalmology.

[9] Erb, C., Barta, A., Bromer, A., Bayer, A.U., Muller-Schaaf and Tjiel, H.J. (1993) Psychiatric Manifestation in Patients with Primary Open Angle Glaucoma. Ophthalmology, 90, 635-639.

[10] Ha, M., Chang, M.H. and Lee, J.H. (2002) Observation on Anxiety and Depression of Glaucoma Patients. Journal of the Korean Ophthalmological Society, 43, 281-289.

[11] Tastan, S., Iyigun, E., Bayer, A. and Acikel, C. (2010) Anxiety, Depression, and Quality of Life in Turkish Patients with Glaucoma. Psychological Reports, 106, 343-357. https://doi.org/10.2466/pr0.106.2.343-357

[12] Zhou, C., Qian, S., Wu, P. and Qiu, C. (2013) Anxiety and Depression in Chinese Patients with Glaucoma: Sociodemographic, Clinical and Self Reported Correlates. Journal of Psychosomatic Research, 75, 75-82. https://doi.org/10.1016/j.jpsychores.2013.03.005

[13] Jayawant, S.S., Bhosle, M.J., Anderson, R.T. and Balkrishnan, R. (2007) Depressive Symptomatology, Medication Persistence and Associated Healthcare Costs in Older Adults with Glaucoma. Journal of Glaucoma, 16, 513-520. https://doi.org/10.1097/IJG.0b013e31804a5ec6

[14] Pappa, C., Hyphantis, T., Pappa, S., Aspiotis, M., Kitsos, G., Psilas, K. and Mavreas, V. (2006) Psychiatric Manifestations and Personality Trait Associated with Compliance with Glaucoma Treatment. Journal of Psychosomatic Research, 61, 609-617. https://doi.org/10.1016/j.jpsychores.2006.03.050

[15] Caceres, V. (2012) Managing Fear and Depression in Glaucoma Patients. American Society of Cataract and Refractive Surgery EyeWorld, 10, 1-4.

https://www.eyeworld.org/article-managing-fear-and-depression

[16] WMA Declaration of Helsinki-Ethical Principles for Medical Research Involving Human Subjects. http://www.wma.net/en/30 publications/10policies/b3 
[17] Kirkwood, B.R. and Sterne, J.A.C. (2003) Medical Statistics. Second Edition, Calculation of Required Sample Size. Blackwell Publishing Limited, Oxford, 413-428.

[18] Zigmond, A.S. and Snaith, R.P. (1983) The Hospital Anxiety and Depression Scale. Acta Psychiatrica Scandinavica, 67, 361-370. https://doi.org/10.1111/j.1600-0447.1983.tb09716.x

[19] Johnson, G.J., Minasssian, D.C., Weale, R.A. and West, S.K. (2012) Epidemiology of Eye Disorders. 3rd Edition, World Scientific Publishers, Singapore. https://doi.org/10.1142/p742

[20] Kanski, J.J. (2006) Clinical Ophthalmology. A Systemic Approach. 6th Edition, Butterworth Heinemann, Philadelphia, 14

[21] Amaryl, M.F. (1967) Genetic Determination of Cup/Disc Ratio of the Optic Nerve. Archives of Ophthalmology, 78, 35-43. https://doi.org/10.1001/archopht.1967.00980030037007

[22] Hodapp, E., Parrish, R.K. and Anderson, D.R. (1993) Clinical Decisions in Glaucoma. Mosby, Maryland Heights, 52-61.

[23] Visual Impairment and Blindness. World Health Organization. http://www.who.int/mediacentre/factsheet/fs28/en/index.html

[24] Uzma, F., Mmunir, H., Asad, R., Surooj, R. and Arshad, S. (2010) Assessment of Anxiety and Depression in Primary Open Angle Glaucoma Patients (a Study of 100 Cases). Pakistan Journal of Ophthalmology, 26, 143-147.

[25] Jampel, H.D., Frick, K.D., Janz, N.K., Wren, P.A., Musch, D.C., Rimal, R. and Lichter, P.R. (2007) Depression and Mood Indicators in Newly Diagnosed Glaucoma Patients. American Journal of Ophthalmology, 144, 238-244. https://doi.org/10.1016/j.ajo.2007.04.048

[26] Mabuchi, F., Yoshimura, K., Kashiwagi, K., Shioe, K., Yamagata, Z., Kamba, S., Lijima, H. and Tsukahara, S. (2008) High Prevalence of Anxiety and Depression in Patients with Primary Open Angle Glaucoma. Journal of Glaucoma, 17, 552-557. https://doi.org/10.1097/IJG.0b013e31816299d4

[27] Mabuchi, F., Yoshimura, K., Kashiwagi, K., Shioe, K., Yamagata, Z., Kamba, S., Lijima, H. and Tsukahara, S. (2012) Risk Factors for Anxiety and Depression in Patients with Glaucoma. British Journal of Ophthalmology, 96, 821-826. https://doi.org/10.1136/bjophthalmol-2011-300910

[28] Wang, S.Y., Singh, K. and Lin, C.S. (2012) Prevalence and Predictors of Depression among Participants with Glaucoma in a Nationally Representative Population Sample. American Journal of Ophthalmology, 154, 436-444.

https://doi.org/10.1016/j.ajo.2012.03.039 


\section{Appendix}

\section{Appendix 1: Ethical Approval from Federal Medical Center, Abeokuta}

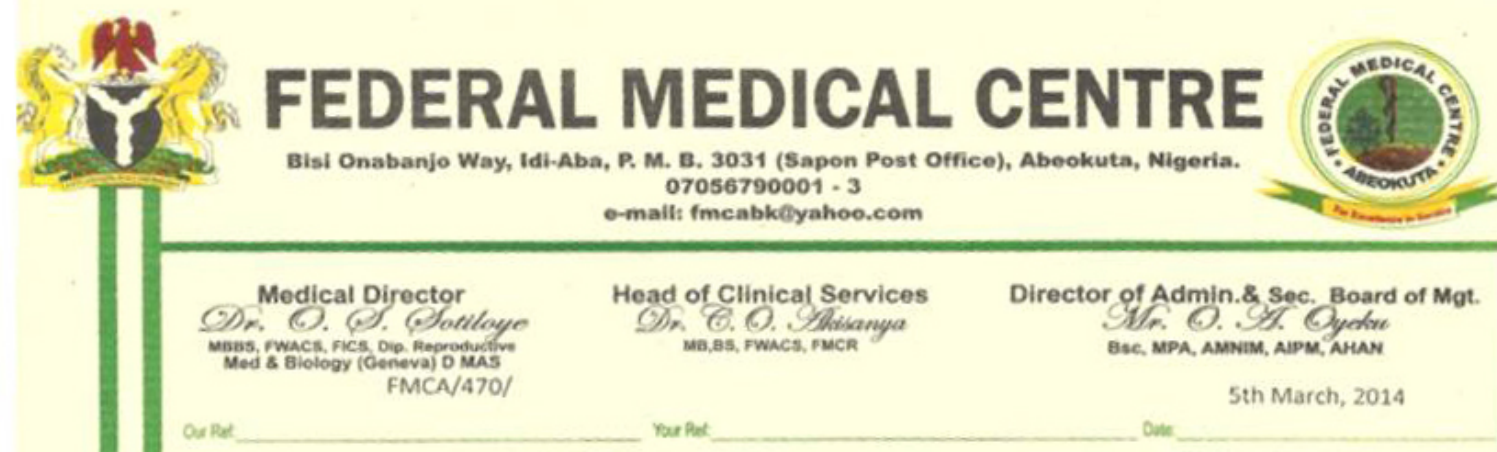

NAME OF PRINCIPAL INVESTIGATOR: Dr. Adaora Chinwendu Okudo

TITLE STUDY:

A COMPARATIVE ANALYSIS OF ANXIETY AND DEPRESSION AMONG GLAUCOMA AND CATARACTOUS PATIENTS ATTENDING A COMMUNITY EYE CARE HOSPITAL IN SOUTHWEST NIGERIA

RESEARCH LOCATION: FEDERAL MEDICAL CENTRE, ABEOKUTA,

PROTOCOL NUMBER: FMCA/238/HREC/04/2014

NREC REG. NUMBER: NREC/08/04/2010

\section{NOTIFICATION OF FULL MEMBER APPROVAL OF RESEARCH PROTOCOL}

This is to inform you that the Federal Medical Centre, Abeokuta Health Research Ethics Committee (HREC) at its sitting on 26th February, 2014 decided to give full membership approval to your research proposal, after necessary reviews and corrections, under the regulations guiding experiments in human subjects.

This approval is for a period of one year from 5th March, 2014 to 4th March, 2015. If there is delay in starting this research, please inform the HREC so that dates of approval can be adjusted accordingly. Note that no activity related to this research may be conducted outside these dates. No changes are permitted in the research without prior approval by HREC.

All forms and questionnaires used in this study must carry the HREC assigned number and the duration of HREC approval.

You are to note further that, the National Code of Health Research Ethics requires you to comply with all institutional guidelines, rules and regulation, to follow trends of the code. Please ensure that any adverse effect from your study is promptly reported to the HREC Federal Medical Centre, Abeokuta.

You are expected to submit a progress report to this Committee every three (3) months from the date of approval. The HREC reserves the right to conduct compliance visits on your research sites without previous notification.

Thank you.

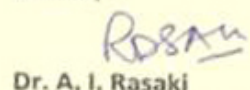

Chairman, Health Research Ethics Committee 


\section{Appendix 2: Ethical Approval from Eye Foundation Health Research Ethics Committee}

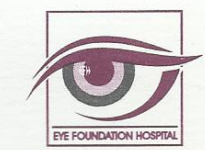

\section{EYE FOUNDATION HOSPITAL}

\section{VITREORETINA}

HASSAN A. 0.

MD, FRCS (Glassow), FRCOphth (U.K.). FWACS

OKONKWO O.N.

MD, FRCS (Ed), FWACS, DRCOphth (U.K)

ODERINLO O.

MD, FRCS (Ed), FWACS, DRCOPhth (U.K)

ANTERIOR SEGMENT

OLUVADI F. O.

HASSAN A. O.

$12^{\text {th }}$ September 2013

GLAUCOMA

OGUNRO A. $O$.

MD, FWACS

HARRIMAN A. S.

MD. DO(ire). MRCOPhth (UK)

OCULOPLASTIC

OLUYADI F. O.

PAEDIATRIC

OPHTHALMOLOGY

ULAIKERE M.

MD, FWACS, FMCOphth

COMMUNITY

OPHTHALMOLOGY

BOGUNJOKO T.

Name of Investigator: Dr. Adaora Chinwendu Okudo

Title of Project : "A comparative analysis of anxiety and depression amongst

glaucoma and cataractous patients in southwestern Nigeria".

Document Reviewed:

- Application

- Proposal

- Questionaire

- Consent form and information sheet for the participants.

Following the review of the Research Ethics Committee of Eye Foundation Hospital. We hereby grant you permission to carry out the research.

CORNEA / REFRACTIVE

SURGERY

OLUYADI F. O.

You will be required to comply with the rules and regulations of the

HASSAN A. 0.

MD, FRCS (Glasgow), FRCOphth (U.K), FW.R A

OPTOMETRISTS

AIREN E. mNOA, O.D, mLVSN

IMAFIDON I. MNOA, O.D.

Mannix U. mNoA, O.D.

FELLOWS

AKANBI T.

MD, FWACS, FMCOphth

ASHAVE A.

MD, FWACS, FMCOphth

ONADIPE 0.

MD, FWACS

BOGUNJOKOT.

MD, DO, MSC (UK), DLSHTM (UK)

SALAU A.
MD, FWACS, FMCOPh

AKINFE A. National Code for Health Research Ethics.

At the end of your study a copy of the final report on the research should be submitted to the institution.

Yours Faithfully,

MD, EMCOphth, M.Sc (epid,)

SUMMIT HEALTHCARE HOSPITAL LTD. Operator of Eye Foundation Hospital

HEAD OFFICE IKEJA: 27B Isaac John Street, G.R.A. Ikeja, P. O. Box 2680, Yaba, Lagos. Tel: 0818200 9943, 08182010165. VICTORIA ISLAND OFFICE: 22, Idowu Taylor Street, Victoria Island, Lagos. Tel: +234 1736 0578, 07028194218 ABUJA OFFICE: Plot 648, Franca Afegbua Crescent off Samuel Jereton Mariere Road Beside Bestway Luxury Suite Zone E, Apo Legislative Quarters Abuja. Tel: +234 98700183

OGUN STATE OFFICE: Old Lagos-Ore-Benin Road llese/ljebu Imushin Ogun State. Tel: $+23417360201,08058950508$ 


\section{Appendix 3: Patient Information Sheet}

\section{Title of research project:}

A comparative analysis of anxiety and depression among glaucoma and cataractous patients in southwest nigeria.

Researcher: Dr. adaora chinwendu okudo

You are invited to take part in a research study. Before you make a final decision to participate in the study, it is necessary for you to understand why the research is being done and what it will involve. I will read the following information to you about this study. If anything is unclear, you would like more information, please do not hesitate to ask.

\section{What is the purpose of the study?}

Glaucoma and cataract are the leading causes of blindness worldwide. A lot of anxiety and depression has been found amongst patients with Glaucoma because of fear of having an irreversible disease that can lead to blindness, fear of surgery and using eyedrops for a long time. Hence we want to carry out a study to observe the burden of anxiety and depression amongst our glaucoma patients and identify patients at risk of these problems in other to plan strategies to deal with these issues. We are using patients that have cataract as our controls for this study.

\section{What is involved in the study?}

During the study a private one on one interview will be held between you and the interviewer or research assistant and you will be asked questions concerning these issues. You will be required to choose options that most applies to you and your situation.

Please be sure to answer each question, taking as much time as you need. In order for this survey to improve our knowledge about anxiety and depression in glaucoma patients your answers must be as accurate and candid as possible.

Please remember that all your data will be kept as strictly confidential.

\section{Do I have to take part in the study?}

It is up to you to decide and sign a consent form. If you do not agree, it will not influence your further examination and treatments. In addition you have a right to withdraw from the study at any time and do not need to explain the reason. 


\section{Appendix 4: Informed Consent Form}

\section{Title of research project:}

A comparative analysis of anxiety and depression among glaucoma and cataractous patients in southwest nigeria.

\section{Researcher:}

Dr. adaora chinwendu okudo

1) I confirm that I have read the information sheet for the above study. I have had the opportunity to consider the information, ask questions and have had these answered satisfactorily.

2) I understand that my participation is voluntary and that I am free to withdraw at any time without giving any reason, without my right being affected.

3) I understand that I can at any time ask for access to the information I provide and I can also request the destruction of that information if I wish.

4) I understand that I will not be identified or be identifiable in any report subsequently produced in any report subsequently produced by the researcher.

5) I accept that taking part in the study is voluntary.

6) I agree to take part in the study.

Participants name:

Date: signature: or Thumb print

Researchers name: Dr. Adaora C Okudo

Researchers Email: adaoraokafor@yahoo.com

Appendix 5: Log Mar Conversion of Snellen's Visual Acuity

\begin{tabular}{ll}
\hline Snellen's Visual Acuity Notation & LogMAR \\
\hline $6 / 6$ & 0 \\
$6 / 9$ & 0.18 \\
$6 / 12$ & 0.3 \\
$6 / 18$ & 0.5 \\
$6 / 24$ & 0.6 \\
$6 / 36$ & 0.7 \\
$6 / 60$ & 1.0 \\
Counting fingers - 6/600 & 2.0 \\
Hand movement - 6/1200 & 2.3 \\
Light perception $-6 / 2400$ & 2.6 \\
No light perception $-6 / 4800$ & 2.9
\end{tabular}




\section{Appendix 6: Questionnaire}

\section{Sociodemographic and Clinical Characteristic Questionnaire}

1. Name:

2. Hospital No:

3. Serial No:

4. Age:

5. Sex: male female

6. Address:

7. Education

- Nil

- Completed primary

- Completed secondary

- Completed tertiary

8. Employment status

- Employed

- Unemployed

- Self employed

- Retired

9. Occupation:

10. Monthly Income:

- $<10,000$

- 10,000 - 20,000

- 20,000 - 50,000

- 50,000

11. Marital status:

- Single

- Married

- Widowed

- Separated/Divorced

12. Religion:

- Christian

- Muslim

- African tradition

- Others

13. Are you aware you have glaucoma: Yes No

14. How long have you had glaucoma? Months Years

b. Family history of glaucoma? Yes No

c. Family history of blindness? Yes No

15. Treatment you have received Eyedrops Tablets Surgery

16. Names of eyedrops:

17. No of drop

Habitual VA RE: habitual VA LE: 
CDR RE: CDR LE:

MD RE: MD LE:

VF DEFECT: MILD MODERATE SEVERE

\section{Hospital Anxiety and Depression Questionnaire}

Please tick in the appropriate column if you have had any of these symptoms within the last one week:

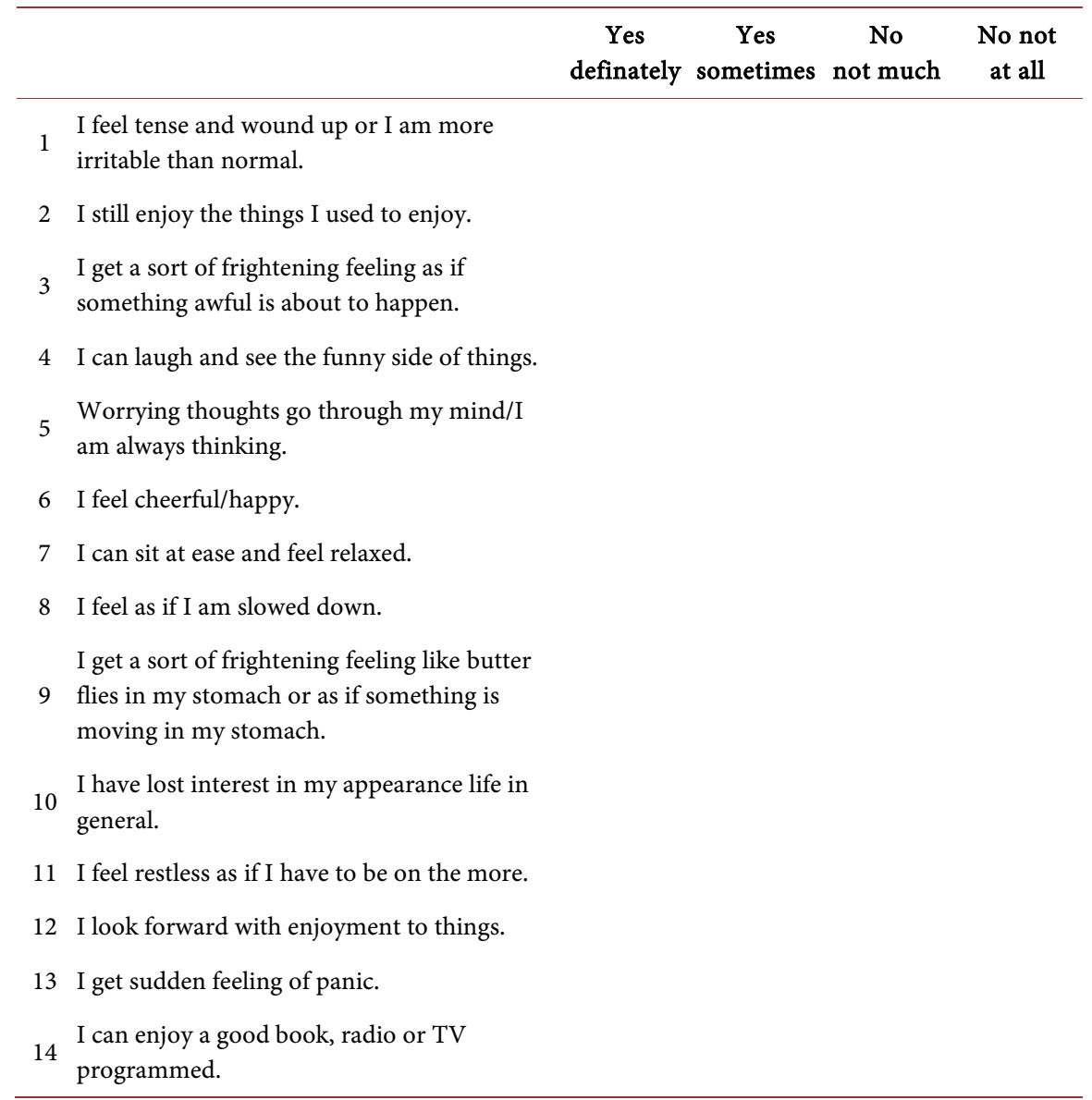

\section{Appendix 7: Scoring of the Hospital Anxiety and Depression Questionnaire}

Questions on Anxiety are questions 1, 3, 5, 7, 9, 10, 13.

Questions on depression are questions 2, 4, 6, 8, 11, 12, 14 .

Scoring 3 for column 1, 2 for column 2, 1 for column 3, 0 for column 4 .

For question 2, 4, 6, 7, 12 and 14 is reversed.

GRADING: 0 - $7=$ Non-case

$$
\begin{aligned}
& 8-10=\text { mild } \\
& 11-14=\text { moderate } \\
& \geq 15=\text { severe }
\end{aligned}
$$

